\title{
NUEVOS ROLES DE LOS DOCENTES EN LA EDUCACIÓN SUPERIOR: HACIA UN NUEVO PERFIL Y MODELO DE COMPETENCIAS CON INTEGRACIÓN DE LAS TIC
}

New roles for teachers in higher education: towards a new profile and competency model with integration of ICT

\section{Luis J. Camacho*}

Resumen: Este artículo presenta una aproximación literaria sobre el debate acerca de cuáles son las competencias necesarias que le permitirán a los docentes del sistema de educación superior superar los obstáculos para dar un salto cualitativo hacia un mayor aprovechamiento de las TIC en los procesos de aprendizaje y enseñanza. Para estos fines el autor inicia con unas reflexiones en torno al contexto actual de la educación superior en el marco de las TIC, por otro lado se realiza un análisis comparativo sobre los distintos enfoques de las competencias, modelo de enseñanza y perfil requerido para los docentes en la educación superior. A manera de conclusión se analizan estrategias para superar los obstáculos que no han permitido una mayor expansión de las TIC a partir de una visión sistémica.

Palabras clave: Competencias TIC, educación superior, educación virtual, perfil docente TIC.

El autor es un mentor y profesor en Empire State College, USA.

Email: luis.camacho@esc.edu 


\begin{abstract}
This article presents a literary approach to the debate about what the necessary skills that will enable teachers of higher education system to overcome obstacles to make a qualitative leap towards a greater use of ICT in the learning and teaching process. To this end the author begins with some reflections on the current context of higher education in the context of ICT, on the other hand a comparative analysis of the different approaches of the competencies required teaching model profile is performed teachers in higher education. In conclusion discusses strategies to overcome the obstacles that have prevented further expansion of ICT from a systemic perspective.
\end{abstract}

Keywords: ICT Skills, Higher Education, Virtual Education, ICT Teaching Profile.

\title{
1. Introducción
}

En los últimos tiempos, han surgido condiciones que han fortalecido el proceso de adopción de las TIC en las aulas y entornos de aprendizaje y esto ha incluido una creciente necesidad de explorar la eficacia en términos de ejecución de los programas y las oportunidades de flexibilidad que brindan las TIC en el proceso educativo (Oliver \& Short, 1996); de igual manera, se integra la capacidad de la tecnología para proporcionar apoyo a los programas educativos adaptados a satisfacer necesidades individuales de los alumnos (Kennedy \& McNaught, 1997) y el creciente uso de internet y la web como herramientas para el acceso a la información y la comunicación (Oliver \& Towers, 2000). 
En los últimos años uno de los medios utilizados para apoyar los programas orientados a mejorar la equidad y el acceso a la educación superior han estado fundamentados en el uso más intensivo de las tecnologías de la información y la comunicación (TIC). Esto ha permitido la expansión del sistema educativo y la posibilidad de mejorar la calidad de vida de los seres humanos, haciendo más plural y democrática la oportunidad de formarse en el nivel avanzado.

No obstante la necesidad de incrementar los ambientes virtuales y tecnológicos en el proceso educativo, se observa un deficiente nivel de competencias en el uso de las tecnologías de información y comunicación en los docentes universitarios que afecta el proceso educativo. Esta situación impide el desarrollo de la enseñanza virtual y a distancia como forma de mejorar la interacción y colaboración entre profesores y alumnos.

El presente artículo presenta una aproximación literaria en torno al debate sobre cuáles son las competencias necesarias que les permitirán a los docentes del sistema de educación superior superar los obstáculos para dar el salto cualitativo hacia un mayor aprovechamiento de las TIC en los procesos de aprendizaje y enseñanza. Para estos fines el autor inicia con unas reflexiones en torno al contexto actual de la educación superior en el marco de las TIC, por otro lado se realiza un análisis comparativo sobre los distintos enfoques de las competencias requeridas para los docentes en la educación superior y según los modelos propuestos en torno a las TIC, finalmente, se presentan estrategias para superar los obstáculos que no han permitido una mayor expansión de las TIC; a partir de una visión sistémica. 


\section{Contexto de la Educación Superior en el marco de las TIC}

La educación superior ha venido recibiendo el impacto de la transformación a partir de la expansión de los conocimientos y saberes, pero de manera imperativa con la implementación de las tecnologías de la información y la comunicación dentro del proceso educativo, se ha generado un cambio dentro de las pedagogías y en la educación a distancia. Por lo tanto, la expansión del conocimiento está facilitando el fortalecimiento del nuevo paradigma educativo y ha contribuido a la generación de una nueva organización universitaria en sus aspectos institucionales, económicos, también en lo relacionado a cobertura y asuntos pedagógicos, una de cuyas manifestaciones está dada por el aumento de la despresencialización de la educación con la introducción de nuevas modalidades de comunicación.

Estos avances en el ámbito tecnológico han sido propulsores de que los profesores y estudiantes tengan la oportunidad de acceder a una mayor cantidad de información de forma rápida, precisa y fiable y, por tanto, las instituciones de educación superior tienen la obligatoriedad de preparar a sus profesores y estudiantes, no solo para acceder a la información, sino también para saber "crear" conocimientos basados en dicha información. Por consiguiente, ante estos constantes cambios dentro de los entornos tecnológicos, y su repercusión en el ámbito educativo, es necesario que el cuerpo docente de todas las instituciones de educación superior se mantengan en actualización permanente, si se quiere una formación de alta calidad y que se le dé respuestas a los cambios en el mundo.

En la actualidad, los docentes deben estar preparados para ofertar a sus estudiantes una forma de aprendizaje basada en las tecnologías de información y comunicación, capacidades que deben ser integradas en el proceso de aprendizaje de los estudiantes, y que forman parte integral del catálogo de competencias profesionales básicas de un profesor. Por tal razón, los docentes necesitan estas 
competencias para empoderar a los estudiantes con las ventajas que les aportan las TIC y que puedan enseñar de manera eficaz las asignaturas exigidas, integrando al mismo tiempo en su enseñanza conceptos y habilidades de estas (Unesco, 2008).

\subsection{EDUCACIÓN A DISTANCIA Y EDUCACIÓN VIRTUAL}

A través de los tiempos se ha demostrado que la educación a distancia posee la capacidad de facilitar educación formal, y no formal a extensos sectores de las sociedades del mundo, que por innumerables razones no tienen acceso a ella de manera presencial. Esta oferta educativa diferenciadora ha sido la gran oportunidad para que muchas personas no vieran sus sueños y deseos de estudiar tronchados; uno porque en términos horarios la educación presencial no les favorecía, dos porque las distancias entre el hogar y los planteles escolares son extremas y, finalmente, porque la obligatoriedad de trabajar para poder subsistir no se los permitían. (Padula, 2003).

Por ser una modalidad educativa que se ajusta a las necesidades de los estudiantes, la educación a distancia ha presentado un significativo crecimiento en los últimos años, tanto en las titulaciones como en la oferta académica, llevando a que se sumen una cantidad importante de instituciones educativas, especialmente universitarias. Como consecuencia de esto, una serie de características surgen en la educación a distancia que la diferencian de la educación presencial, como son: a) la autonomía e independencia del alumno en el proceso de aprendizaje; b) el carácter práctico que imprimen los estudiantes a los aprendizajes al ser que muchos de ellos desarrollan actividades laborales con vinculación a sus propios estudios; $\mathrm{y}, \mathrm{c})$ incremento de la responsabilidad y el compromiso del estudiante con el proceso de aprendizaje. (Suárez \& Anaya, 2004).

Según Alarcón (2012) aunque existen paradigmas que se oponen a la viabilidad de la educación a distancia, esta ha podido superar las fronteras y cumplir con su cometido de democratización de la 
educación en todos los ámbitos sociales ya que su enorme potencial admite un avance y desarrollo beneficiando gran parte de la población que solo tiene este modelo educativo como posibilidad de desarrollo personal y académico. Pero, aunque es el modelo educativo que permite la inclusión de amplios sectores sociales, la educación a distancia demanda la existencia de condiciones mínimas, pero vitales, para aquellos que optan por esta. Es necesario contar con un estudiante a distancia que sepa leer en forma comprensiva y expresarse por escrito, al mismo tiempo ser diestro en la escritura a mano alzada, mecanografiada o digital, y tener acceso a aparatos de reproducción de audio y/o video o, en determinados casos, a computadoras personales e internet. (Padula, 2003).

Un hecho relevante dentro del proceso de desarrollo de la educación a distancia lo fue el que se produjo en la Conferencia Mundial de la Unesco de 2009, la cual versó sobre el tema "La nueva dinámica de la educación superior y la investigación para el cambio social y el desarrollo". Uno de los grandes planteamientos que allí se esbozaron fue el significado de la tecnología como elemento fundamental para mejorar la calidad de la educación superior y ajustarla a las exigencias de las sociedades del conocimiento del siglo XXI (Unesco, 2009).

En este evento se evaluaron los avances de los acuerdos cumplidos durante una década y lo que habría que hacer para alcanzar las prioridades: la internacionalización, la regionalización y la globalización; el acceso, la equidad y la calidad; la formación, la investigación y la innovación en la educación superior. Allí se hizo énfasis en la importancia estratégica del:

"... aprendizaje abierto y a distancia, y el uso de las TIC [que] ofrecen oportunidades de ampliar el acceso a la educación de calidad, en particular cuando los recursos educativos abiertos son compartidos fácilmente entre varios países y establecimientos de enseñanza superior." (p. 3). 
La humanidad ha sido partícipe de un cambio de paradigma en las áreas de la tecnología y la economía a partir de la década de los ochenta. Estos cambios se han expresado en las ciencias computacionales creando consecuentemente nuevas formas de producción y clasificaciones sociales, entre las que está presente la gestación de una nueva educación. (Rama, 2009; Casas, 2005).

Como consecuencia de todo esto se ha constatado un cambio en las formas de trasmisión del conocimiento, pero inminentemente una alteración de la educación a distancia cuando esta se involucra con la virtualización e intercambio del conocimiento por medios virtuales. Es por esto que la base del fortalecimiento del nuevo paradigma educativo, especialmente cuando este se inclina hacia una nueva organización universitaria en sus aspectos institucionales, económicos, de cobertura y pedagógicos, es el crecimiento de la educación no presencial por medio de modalidades de comunicación. (Rama, 2012).

Estos avances en el ámbito tecnológico han sido propulsores de que la ciudadanía tenga la oportunidad de acceder a una mayor cantidad de información de forma rápida, precisa y fiable. Sin lugar a dudas, todo este cúmulo de herramientas y proceso debe llevarles a una preparación imprescindible para su futuro en la sociedad. (Palomo, Ruiz \& Sánchez, 2006).

No cabe dudas de que las instituciones de educación superior deben jugar un papel preponderante para crear las condiciones necesarias que lleven a las referidas sociedades tradicionales a tener que asumir el reto de un cambio hacia la sociedad de la información y hacia la sociedad del conocimiento. Para avanzar hacia tales objetivos, Tünnermann (2000) señala:

La médula del problema radica en que nos encontramos en una etapa de transición y por lo mismo crítica, entre la educación superior elitista y la educación superior masiva. Las actuales estructuras académicas responden a la educación superior elitista; por lo mismo, les es imposible, 
sin una profunda transformación, hacer frente al fenómeno de masificación. Son, en general, demasiado rígidas, poco diversificadas, y carentes de adecuados canales de comunicación entre sus distintas modalidades y con el mundo de la producción y del trabajo. La homogeneidad de sus programas no les permite atender la amplia gama de habilidades, intereses y motivaciones de una población estudiantil cada vez más extensa y heterogénea; su excesiva compartimentalización contradice la naturaleza esencialmente interdisciplinaria del conocimiento moderno; su apego a los sistemas formales les impide servir con eficacia los propósitos de la educación permanente. (2000: 100-101).

Lógico es decir que para estructurar estos conceptos se debe contar con una herramienta fundamental, la innovación. En este sentido Albornoz (2002), en Casas \& Stojanovic (2013), afirma que la innovación se convierte en la base de la sociedad del conocimiento, además de ser uno de los motores de la globalización y que para que el desarrollo social y humano sea sostenible la innovación debe regirse por valores éticos y morales.

A partir de la gran integración de las TIC se ha creado en la educación superior, lo que se denomina "e-learning", y se ha evidenciado cómo se ha llegado a una tercera década de cambio en la forma de diseñar los programas y cursos, de igual manera como son entregados a los estudiantes. Ha sido notable la transformación que ha producido el e-learning y las tecnologías aplicadas a la educación superior (Kanuka \& Kelland, 2008).

Es preciso puntualizar que el uso de tecnología en la educación contemporánea, la cual potencia un aprendizaje autónomo y formación humanista ha traído consigo una gran cantidad de ofertas educativas que requieren una serie de criterios que permitan llegar a un análisis objetivo de las diferentes opciones y características necesarias dentro del estudiantado y el profesorado. La educación virtual integra las viejas tecnologías de la literatura impresa con las modernas tecnologías de la información y comunicación (Méndez-Estrada \& Monge, 2006). 
La integración de las tecnologías de información y comunicación en la educación tiene como finalidad, mejorar la calidad de la misma pero a la vez reducir las desigualdades. Esta conceptualización debe llevar a la conclusión de que los planes de estudios actuales deben ser más relevantes, por lo tanto, se debe adaptar el contenido, los métodos y las instituciones educativas a las situaciones sociales, culturales, lingüísticas y geográficas específicas de la sociedad e implementar las TIC y sus recursos para elevar los resultados de la educación superior (Katz \& Hilber, 2003).

Pero lo cierto es que aunque se ha avanzado mucho con la calidad en la educación virtual también se observa una proliferación y producción de programas de educación a distancia carentes de una profunda evaluación. Esta realidad debe llevar a la identificación de algunos criterios que puedan ser utilizados como filtros para contrastar que son propuestas educativas confiables y de calidad en la práctica (Fainholc, 2002).

Entonces, la renovación universitaria se bate en este conflictivo y complejo panorama, donde se tienen necesidades de transformación pero obstáculos que constriñen la misma. Por lo tanto, resulta imprescindible incorporar la innovación en su moderna acepción, como el instrumento ideal para gestionar el cambio dentro de las instituciones de educación superior, provocando que estas puedan adquirir una trascendente y decisiva transformación que les permita llevar el conocimiento y la modernización a las sociedades donde coexiste (Casas, 2005).

Cuando se habla de educación virtual se debe reconocer que existen diferentes niveles de virtualización dentro de las instituciones de educación superior que han asumido este modelo educativo, por lo que se puede fluctuar desde lo que se denomina virtualidad, como una forma complementaria de la presencialidad, hasta llegar a la virtualización total (Sangrà, 2002). En este mismo contexto, este autor identifica tres grados de virtualización dentro de las instituciones de educación superior, que son: 
a) Según la iniciativa: universidad presencial que introduce elementos de virtualidad en su dinámica educativa, universidad presencial con extensión universitaria virtual y espacios compartidos de cursos virtuales que ofrecen las universidades presenciales, universidad virtual adosada a la universidad tradicional, universidad virtual como organización virtual y espacios virtuales interuniversitarios comunes.

b) Según la oferta: temática y volumen.

c) Según la tecnología de distribución (delivery system): modelos principalmente síncronos, modelos parcialmente asíncronos y modelos esencialmente asíncronos.

Para Uñantes, Reynoso y Brescia (2001) e-learning se puede definir como "un tipo de enseñanza a través del uso de la tecnología informática y de redes de datos involucrando la distribución de contenidos pedagógicos a través de internet, intranets/extranets, audios y video, enlaces y transportes satelitales, TV interactiva o CD-ROM". Aunque Padula (2002) no solo se queda en la mera integración de los elementos tecnológicos, este enfatiza que la educación virtual requiere tutores que estén altamente familiarizados con las TIC's de manera tal que puedan crear un ambiente en el que los estudiantes, sus necesidades y su asesoría converjan en un espacio virtual, donde la flexibilidad en cuanto al manejo de los tiempos, la no presencialidad, el seguimiento a los planes de estudios desde cualquier parte y el alto grado de autonomía afianza a la educación a distancia como una opción apropiada para los tiempos actuales.

Al romper con los paradigmas de la educación tradicional, muchas son las interpretaciones que se pueden tener cuando se habla de la calidad de la educación virtual. Para Murcia (2004) muchos hablan de la calidad de la educación pero educar no es solo enseñar, instruir o informar, educar es ante todo formar, transformar, comunicar, socializar, al menos en tres procesos: económico o rentable, 
valorativo o cualitativo y socializante o comunicativo; esto implica una verdadera educación [virtual] o formación integral en la producción y apropiación social del conocimiento, dentro del mismo proceso de investigación humanística y tecnológica.

$\mathrm{Al}$ referirse Murcia (2004) al concepto de "campus virtual" este lo concibe en la forma en que se aplican a la educación superior las tecnologías de la información y la comunicación, por lo tanto, cuando se crea un tipo o modelo de enseñanza que puede ser accesible desde cualquier lugar y a cualquier hora, mediante una conexión que permite la interacción diferida o en tiempo real, en la comunidad educativa virtual. Por lo tanto, se hace imprescindible que los docentes adquieran las capacidades de estas nuevas tecnologías educativas de manera que se pueda provocar el cambio que contribuya a la transformación de las prácticas pedagógicas, permitiendo la interactividad y la aplicación de nuevos medios, con la posibilidad de utilizar y cambiar los sistemas educativos tradicionales.

Uno de los elementos más importantes que se pueden identificar con relación a la educación virtual es que a través de ambientes virtuales, recursos tecnológicos digitales a través de software específicos y al interior de redes y plataformas de aprendizaje virtuales, esta ha traído consigo la solución de una de las problemáticas más discutidas, pero menos atendidas a través de los tiempos dentro de la educación superior: la inclusión educativa. A través de la expansión de la educación basada en las TIC ha nacido un nuevo enfoque educativo, donde por medio de la informática, los ambientes virtuales de aprendizaje, las redes virtuales de aprendizaje y los recursos digitales se atienden a amplios sectores por siempre desatendidos. (Rama, 2012). 


\section{Análisis comparativo sobre competencias en educación superior}

De acuerdo con Montmollin (1984), competencia se puede definir como: "Conjunto estabilizado de saberes y saber-hacer, de conductas tipo, de procedimientos estándares, de tipos de razonamiento, que se pueden poner en práctica sin nuevo aprendizaje". Asimismo, Hayes (1985) define competencia como "la capacidad de usar el conocimiento y las destrezas relacionadas con productos y procesos $y$, por consiguiente, de actuar eficazmente para alcanzar un objetivo".

Según el análisis presentado por Prieto (1997) sobre los diferentes conceptos que pueden ser identificados con el término competencia y que este ubica dentro del ámbito socio-laboral, el autor no define la competencia como autoridad, haciendo clara alusión a los asuntos o cometidos que dan bajo la competencia directa de un profesional concreto o una figura profesional. En este caso, se está ante la acepción de competencia como atribución o incumbencia, estando ligada a la figura profesional (tareas y funciones) que "engloba el conjunto de realizaciones, resultados, líneas de actuación y consecuciones que se demandan del titular de una profesión u ocupación determinada".

De igual manera ubica la competencia como capacitación, refiriéndose al grado de preparación, saber hacer, conocimientos y pericia de una persona como resultado del aprendizaje. En este caso, la competencia alude directamente a las capacidades y habilidades que una persona debe desarrollar a través de la formación. También podría considerarse en este punto la competencia como cualificación, referida básicamente a la formación necesaria para tener la competencia profesional deseada.

Por último, puede aludirse a la competencia como suficiencia o mínimos clave para el buen hacer competente y competitivo. En este caso, se acotan las realizaciones, resultados, experiencias, logros 
Nuevos roles de los docentes en la educación superior: hacia un nuevo perfil y modelo de competencias con integración de las TIC

de un titular que debe sobrepasar para acceder o mantenerse satisfactoriamente en una ocupación con garantías de solvencia y profesionalidad.

Chiavenato (2002: 307), considera que "mediante el desarrollo de competencias la persona puede asimilar información, aprender habilidades, desarrollar actitudes y comportamientos diferentes y comprender conceptos abstractos". En ese sentido, plantea el desarrollo de competencias basado en un modelo de cuatro etapas: la primera es la de transmisión de informaciones, a través de la cual se pretende aumentar el conocimiento de las personas; la segunda concierne al desarrollo de habilidades y destrezas con el propósito de mejorarlas; la tercera trata sobre el desarrollo de actitudes, con el fin de desarrollar o modificar comportamientos; y, la cuarta, el desarrollo de conceptos, que busca elevar el nivel de abstracción. Este autor entiende que para enfrentar con éxito profesional los desafíos del mundo moderno se requiere desarrollar competencias durables, el conocimiento, la perspectiva y la actitud.

Por su parte, Gallart y Jacinto (1995) presentan su conceptualización de competencia como el "conjunto de saberes puestos en juego por los trabajadores para resolver situaciones concretas de trabajo". De igual modo, LévyLeboyer (2002) afirma que competencia se refiere a "repertorios de comportamientos que algunas personas dominan mejor que otras, lo que las hace eficaces en una situación determinada (...); ponen en práctica, de forma integrada aptitudes, rasgos de personalidad y conocimientos adquiridos".

Según Pereda y Berrocal (2001), las competencias se estructuran en base a los siguientes componentes fundamentales:

- Saber: como conjunto de conocimientos generales y específicos.

- Saber hacer: capacidad de aplicar los conocimientos a la solución de situaciones concretas de trabajo, cada vez menos estructuradas. 
- Sabe restar: asociado a las "competencias blandas"; relaciones interpersonales, capacidad para trabajar con otros, comunicar, liderar.

- Querer hacer: en referencia a la responsabilidad, la iniciativa y el compromiso con el logro de objetivos.

La tendencia hacia una sociedad basada en el conocimiento ha sido aceptada ampliamente y se encuentra consolidada desde hace algún tiempo. Algunos elementos que definen este cambio de paradigma son: una educación centrada en el estudiante, el cambiante papel del educador, una nueva definición de objetivos, el cambio del enfoque de las actividades educativas y en la organización y los resultados del aprendizaje.

\subsection{LAS COMPETENCIAS DOCENTES}

El objetivo primordial de la educación es desarrollar capacidades, pero si estas se orientan de manera única a las realizaciones prácticas se tendría como resultado un modelo instruccional basado en la duplicación de actuaciones mecánicas, que no tienen relación directa con la educación basada en la formación integral del individuo. Entonces, cuando se habla del establecimiento de los objetivos educacionales se debe hacer sobre la base del desarrollo de capacidades, y la forma como estas pueden ser aplicadas de manera funcional a las habilidades propias de cada persona. (Dirección General de Ordenación y Evaluación Educativa de Andalucía, 2012).

Sobre la base de los objetivos educacionales y la integración de diferentes variables dentro del concepto de competencia, Aguilar \& Cepeda (2005) plantearon como definición de competencia lo siguiente:

Un sistema de acción complejo que abarca las habilidades intelectuales, las actitudes y otros elementos no cognitivos, como motivación, valores y emociones, que son adquiridos y desarrollados por los individuos a lo largo de su vida y son indispensables para participar eficazmente en diferentes contextos sociales. (2005: 16). 
Nuevos roles de los docentes en la educación superior: hacia un nuevo perfil y modelo de competencias con integración de las TIC

Según la definición de Escudero (2006) se entiende por competencia docente el "conjunto de valores, creencias y compromisos, conocimientos, capacidades y actitudes que los docentes, tanto a título personal como colectivo (formando parte de grupos de trabajo e instituciones educativas) habrían de adquirir y en las que crecer para aportar su cuota de responsabilidad a garantizar una buena educación para todos" (Escudero, 2006: 34).

Hellriegel y Slocum (2005: 30), presentan cinco tipos de competencias básicas:

1) "Competencias técnicas: capacidad de aplicar métodos, procedimientos y técnicas específicas en un campo especializado;

2) Competencias interpersonales: capacidad individual para dirigir, motivar, resolver conflictos y trabajar con los demás;

3) Competencias de conceptualización: capacidad para percibir un problema, asunto u organización como un todo, así como sus partes interrelacionadas;

4) Competencias de comunicación: capacidad para la emisión y la recepción de información, ideas, opiniones y actitudes; y

5) Competencias de razonamiento crítico: consideración minuciosa de las implicaciones de todos los elementos conocidos de un problema".

Ampliando el tema sobre el concepto de competencias Imbernón, Silva \& Guzmán (2011: 108), después de una revisión de varios autores, plantearon una síntesis que muestra otra serie de elementos a ser tomados en consideración:

en el concepto [competencias] se integra el saber, el saber hacer y el saber ser; tiene relación con la acción, se desarrolla y se actualiza en la acción; está vinculada a un contexto, a una situación dada y facilita la resolución eficaz de situaciones laborales conocidas o inéditas y es educable. 


\subsection{NUEVAS COMPETENCIAS DOCENTES EN LA EDUCACIÓN SUPERIOR}

Ante los desafíos y cambios sustanciales que se enfrentan en la actualidad, especialmente si se parte de la premisa de la inserción en la sociedad de la información y del conocimiento, es imperante formar ciudadanos que sean capaces de descodificar, de resignificar y transformar la información en conocimiento aplicado. Pero de manera especial, si se refiere a la educación superior, que es la que debe dar respuesta a estos retos a través de una práctica educativa que fomente el uso y aplicación de las TIC, se debe garantizar el acceso y uso crítico de la información para la transformación de todos estos elementos en el conocimiento mismo. (Larraz et al., 2013).

Imbernón, Silva \& Guzmán (2011), cuando presentan su estudio sobre las competencias digitales, exponen lo siguiente:

Están relacionadas con "el uso seguro y crítico de las tecnologías de la sociedad de la información (TSI). Se sustenta en el uso de ordenadores para obtener, evaluar almacenar, producir, presentar e intercambiar información y comunicarse y participar en redes de colaboración a través de Internet) (Comisión de las Comunidades Europeas, 2005: 18)". Estudios recientes destacan que los docentes universitarios deben dominar y saber desarrollar dichas competencias para facilitar los procesos de aprendizaje autónomos y significativos. Los profesores, por tanto, también debemos saber, conocer, seleccionar, utilizar, evaluar, perfeccionar y recrear o crear estrategias de intervención didáctica efectivas en un contexto definido por las TIC y con el European Credit Transfer and Accumulation System (ECTS) como telón de fondo. (2005: 109)

Tello (2003) plantea el concepto de competencia informática como: "el conjunto de capacidades adquiridas en el campo informático que posibilitan al sujeto relacionarse con el ordenador de tal manera que sea capaz, además de reconocer e identificar las partes del mismo, cubrir objetivos personales, académicos y/o 
profesionales mediante la utilización de software específico para poder gestionar la información, la comunicación y la resolución de problemas. (2003: 39)

Desde la óptica de la Comisión Mixta CRUE-TIC y REBIUN (2012), el concepto de competencia informática sería el siguiente: "el conjunto de conocimientos, habilidades, disposiciones y conductas que capacitan a los individuos para saber cómo funcionan las TIC, para qué sirven y cómo se pueden utilizar para conseguir objetivos específicos." (2012: 6)

De igual manera, la comisión mixta CRUE-TIC y REBIUN (2012: 6), tratando de separar en cierta medida el concepto de competencia informática con el de competencias informacionales, aporta la siguiente definición: "Las competencias informacionales son el conjunto de conocimientos, habilidades, actitudes y conductas que capacitan a los individuos para reconocer cuándo necesitan información, dónde localizarla, cómo evaluar su idoneidad y darle el uso adecuado de acuerdo con el problema que se les plantea".

\section{Los nuevos roles de los docentes en la educación superior: hacia un nuevo perfil y modelo de compe- tencias propuestos con integración de las TIC}

Es necesario entender que existe la necesidad dentro del ámbito de la educación superior para el desarrollo profesional que pueda preparar los docentes para la educación virtual, y que producto de esto existen muchos modelos que han sido implementados con diferentes enfoques y elementos, fundamentalmente la tecnología, pedagogía y de contenidos. Pero, aunque se conoce y reconoce la necesidad de formación de estos docentes una parte importante de ellos se integran por primera vez al aula virtual sin haber sido capacitados creándose un impacto negativo tanto para el profesorado como para los estudiantes (Abel, 2005). 
Reigeluth (2012), plantea un sumario de los roles que deben existir a partir del nuevo paradigma de la instrucción:

Los nuevos roles para los docentes incluyen: diseñar el trabajo de los estudiantes, facilitar el proceso de aprendizaje y ser un mentor. El nuevo rol para los estudiantes incluye: trabajar, aprender de manera auto dirigido y enseñar. Mientras más teoría instruccional ha sido generada para guiar el diseño de los nuevos paradigmas de instrucción, más falta por aprender. Necesitamos aprender [así] como señalar mejor las fuertes bases emocionales del aprendizaje, fomentar el desarrollo social y emocional y promover el desarrollo de actitudes positivas, los valores, la moraly la ética, entre otras cosas.

El crecimiento del mercado de la educación virtual es extraordinario, tanto que existe en este momento una presión real para que se puedan ofertar nuevos y más amplios programas educativos y profesionales. Pero, a medida que crece el mercado en este sentido crece la resistencia de parte de los docentes para involucrarse en la instrucción virtual. Por lo tanto, uno de los grandes retos a ser encarados por las instituciones de educación superior lo es sin dudas obtener la aprobación de sus docentes para ser capacitados con este modelo educativo.

En este momento las instituciones de educación superior están compelidas a trabajar para superar esta situación, pero de manera clara los miembros de las facultades se sienten insatisfechos con el nivel de soporte institucional para la instrucción virtual. Esto tiene varias explicaciones: por un lado, porque los docentes que no han enseñado dentro del modelo virtual desarrollan una percepción negativa de la instrucción en línea, especialmente porque perciben que tienen una falta de desarrollo profesional como la mayor barrera para desarrollar e implementar programas en línea $Y$ de igual forma, porque al estar las instituciones de educación superior mejorando o haciendo crecer sus programas en línea esto les impone una presión por el conocimiento que combinada con su ausencia de elementos básicos de las TIC incrementan estas barreras. (Heather, 2012) 
Por medio de la integración y uso de las tecnologías de información y comunicación en el ámbito educativo se ha creado una brecha de oportunidad para los estudiantes en relación a poder adquirir amplias capacidades en el uso de estas; lógicamente, existe una figura que es la más importantes en el proceso de ayuda a estos estudiantes, el docente, quien no solo es el responsable de coadyuvar en el proceso de aprendizaje sino que es el que tiene sobre sus hombros la tarea de diseñar los instrumentos a ser compartidos para el aprendizaje como el entorno propicio en el aula integre y facilite el uso de las TIC.

Esto implica, de manera insoslayable, que todos los docentes se deben involucrar en un programa de desarrollo profesional en el uso de las TIC con la finalidad de estar preparados para compartir con sus estudiantes estas oportunidades de aprendizaje. Las instituciones de educación deben mantener dentro de sus planteles, virtuales o no, docentes con profundas competencias en tecnologías de información y comunicación con la finalidad de enseñar de manera eficaz las asignaturas exigidas e integrando al mismo tiempo en su enseñanza conceptos y habilidades de estas. (Waheed, 2008).

Por otro lado, la integración del uso de las TIC dentro de los programas docentes demanda nuevas pedagogías y nuevos planteamientos en la formación docente. En tal sentido, estos tendrán una fuerte dependencia de la capacidad para estructurar el ambiente de aprendizaje de forma no tradicional, y en cómo unificar las TIC con nuevas pedagogías y fomentar clases dinámicas en el plano social, estimulando la interacción cooperativa, el aprendizaje colaborativo y el trabajo en grupo. Para lograr todo esto, es indispensable que el docente adquiera competencias para manejar la clase. (Unesco, 2008).

Una lista de roles de los e-tutores fueron provistas por Barker (2002: 7) que incluyen el “...pastoreo de estudiantes” en relación a asesorarlos acerca de sus carreras y elecciones de cursos, haciendo las asignaciones a los estudiantes y proveyéndoles retroalimentación 
en los materiales suministrados". De igual manera, Barker incluye el rol de moderador de conferencias, actuando como mentores a los colegas menos experimentados y haciendo un control de calidad a otros e-tutores de trabajo.

La importancia del docente dentro de la educación virtual es fundamental, este actúa como un facilitador, el cual con su vasto conocimiento y experiencias contribuye a la creación del aprendizaje y dirige las discusiones a los puntos críticos. De igual manera, el docente como tutor pregunta y responde al estudiante, aleja los análisis fuera de lugar y hace las síntesis que permiten desarrollar temas de interés para el área de aprendizaje (Ryan et al., 2000).

En este sentido Lentell (2003) aporta lo siguiente: "los tutores facilitan y guían el aprendizaje de sus estudiantes de manera tal que el estudiante adquiere el conocimiento y el entendimiento. Para lograr esto, el tutor desarrolla y práctica una multitud de habilidades y estrategias". (2003: 67). Es importante resaltar que existen dos grandes áreas de soporte del tutor:

1) La académica, que es la que soporta al estudiante con los elementos cognitivos, intelectuales y conocimientos; y

2) La no académica, que es el soporte a los estudiantes en el plano afectivo y organizacional de sus estudios (Simpson, 2002).

En búsqueda de la enseñanza innovadora dentro de la educación a distancia surge el paradigma de la educación multimedia, el cual se convierte de manera inmediata en un área y ciencia aplicada que incide de manera directa en el proceso de evolución y desarrollo de la enseñanza-aprendizaje. Todo este cambio impacta y afecta la perspectiva contemporánea de la educación superior, de manera muy especial en la forma en la que se puede acceder al conocimiento y la realidad, además de la forma de aprender y educar (Navarro, 2009, citado por Vales, 2009). Este tipo de educación conduce a una educación mediatizada, que en palabras de Padula 
Nuevos roles de los docentes en la educación superior: hacia un nuevo perfil y modelo de competencias con integración de las TIC

(2004) no es más que el proceso de comunicación entre maestroalumno en el cual existen distintos medios indirectos que la hacen posible - sean gráficos, audiovisuales, auditivos o informáticos.

Sin lugar a dudas las TIC son el referente fundamental para llegar a la virtualización de la educación, especialmente cuando se refiere a los instrumentos y herramientas que sirven de ayuda a los que tienen que crear los contenidos de enseñanza, como a los alumnos para facilitar el logro de sus objetivos de aprendizaje (Cabero \& Aguaded, 2003, en Imbernon et al., 2011). En relación a este punto, Zamora (2002) indica que el desarrollo de las competencias informáticas dentro de las instituciones formadoras de docentes es fundamental y relevante para optimizar el proceso de enseñanzaaprendizaje-evaluación, especialmente cuando se integran recursos como las WebQuest, que constituyen una actividad didáctica basada en elementos constructivistas del aprendizaje (Adell, 2004).

La integración del internet en las actividades normales del individuo no solo es importante por la obtención de información constante de cualquier tipo, lo más importe es que se ha convertido en una herramienta tecnológica que se ajusta de manera perfecta al proceso de culturización y educación del individuo en cualquier nivel. Pero si se enfoca el uso del internet al ámbito de la educación, ya son muchas las aplicaciones que se tienen a la mano que han ayudado y ayudan en el proceso de transferencia del conocimiento, por ejemplo: e-learning, b-learning, educación en línea, educación semipresencial, educación virtual, educación en equipos móviles, donde cada una de ellas induce a continuar profundizando los estudios y aplicaciones de las TIC en el proceso de educación.

Sobre la base de estas premisas, Silvio (2006) enfatiza que es inminente incrementar la calidad en la educación virtual y a distancia, al igual que su mejoramiento permanente, pero más aún es hacerlo procurando que esa educación alcance la mayor equidad y pertinencia social. Al tener la educación en línea una gran cantidad de características multidimensionales de igual manera demanda una 
gran cantidad de competencias, obligando a las instituciones de educación superior, pero de manera especial a los docentes, a repensar su rol y competencias dentro de la educación tradicional. La educación superior ha tenido que asumir diferentes procesos tecnológicos, especialmente en la implementación y el uso de las TIC, que interrelacionadas con las exigencias de los innovadores sistemas de administración del aprendizaje han reconstruido y redimensionado el ámbito general de la educación superior (Amirault and Visser 2009; Harasim 2006; Laurillard 2008; Tapscott and Williams 2010; Abdous and Yoshimura 2010).

La gran brecha que se ha mantenido entre la enseñanza y el aprendizaje cara a cara y la enseñanza en línea se ha venido cerrando día tras día, de manera sostenida. El gran auge y el incremento de las diversas ofertas educativas que integran una gran cantidad de métodos educacionales, tales como: la educación a distancia, la educación semipresencial y la educación en línea han permitido reconsiderar los roles y las competencias de los docentes, y la forma como estos deben asumir su carga, el tiempo de enseñanza y su presencia con los estudiantes (Means et al., 2009; Parsad and Lewis 2008; Vaughan 2007).

Esta nueva forma de entender el rol de los docentes y sus competencias ha permitido una transformación integral donde una cantidad importante de nuevos enfoques dentro del ambiente educativo están presentes, y que no eran entendidos ni asumidos algunos años atrás. En la actualidad, la integración de tecnología en el proceso de enseñanza-aprendizaje, el crecimiento exponencial de la demanda de la oferta en línea, el crecimiento de la demanda de estudiantes no tradicionales y los ajustes que se han tenido que hacer a los currículos de las instituciones de educación superior son elementos fundamentales para poder satisfacer la demanda de educación de manera muy especial en la educación superior. (McShane, 2004). 
En los últimos años se han mostrado muchos estudios tendentes a identificar y llevar luz sobre el tema de las competencias asociadas a la educación en línea, aunque para Bawane \& Spector (2009) las competencias para la enseñanza en línea no son distintas a la enseñanza presencial. No obstante, por medio de estos estudios se han matizado aún más algunas imprecisiones o contraposiciones relacionadas [con] estas competencias docentes, de igual manera han ayudado a mejorar el entendimiento de los retos institucionales y personales asociados con la educación en línea (Briggs, 2005).

La literatura sobre la enseñanza en línea es abundante, pero pocos estudios han presentado de manera sistemática los roles y competencias asociadas a la enseñanza en línea. Paulsen (1995) propuso una clasificación estructurada acerca de tres roles interrelacionados: el organizacional, el social y el intelectual.

Siguiendo con el estudio de Paulsen (1995), el rol organizacional intenta agrupar una agenda que clarifique los objetivos de la discusión y que pueda calendarizar y resaltar las reglas de procedimiento y las normas en la toma de decisiones. El rol social tiene como objetivo la creación de un ambiente social amigable, favorable para el aprendizaje en el cual los miembros de la facultad les puedan dar la bienvenida a los estudiantes y que les estimules a participar mediante un modelo de rol y proveyéndoles retroalimentación constructiva de una manera responsable y amigable. El rol intelectual intenta mantener la discusión enfocada en los puntos cruciales, mientras se suman ideas y se estimula a los estudiantes a expandir y construir en los comentarios que son generados.

En ese contexto, Berge (1995) presentó un modelo organizado alrededor de cuatro dimensiones: la dimensión pedagógica, dimensión social, dimensión de gestión y dimensión técnica. Como se puede apreciar, la única diferencia con el modelo de Paulsen (1995) es la integración del rol técnico, el cual ha sido integrado con la intensión de reforzar las habilidades técnicas de los docentes. 
Trabajando sobre los resultados de un taller que involucraba practicantes e investigadores de Estados Unidos, Inglaterra y Europa Goodyear, Salmon \& Spector (2001) trataron de entender y categorizar las competencias de enseñanza en línea, e intentó responder algunas preguntas, tales como la forma de contratar, entrenar, evaluar y certificar los docentes como profesores de cursos en línea. Goodyear et al., pudieron identificar ocho diferentes roles (facilitador del proceso, asesor/consejero, investigador, facilitador de contenido, tecnólogo, diseñador y gerente/administrador) asociado con las tareas específicas ejecutadas por los docentes.

Utilizando el enfoque de análisis de patrón, Coppola et al. (2002) presentaron tres roles que los docentes deberían desarrollar cuando se encuentran en el proceso de transición de educación "cara a cara" a la educación "virtual". Primero, el desarrollo de un rol cognitivo, el cual se refiere al proceso de aprendizaje mental, almacenamiento de información y pensamiento; segundo, el desarrollo del rol afectivo, el cual se refiere a la relación instructor y estudiante, y requiere que los docentes aprendan nuevas herramientas para expresar sus emociones; y, tercero, el desarrollo del rol de gestión, el cual se refiere a la negociación con la clase y la administración del curso, ya que los cursos en línea a veces requieren la adopción de nuevas formas de monitorear a los estudiantes.

Por su parte, Mishra \& Koehler (2006) ofrecieron interesantes elementos de la integración tecnológica en la educación a los profesores y el desarrollo de los docentes a partir de una perspectiva teórica, y tomando como referencia los planteamientos de Shulman (1987) con relación a los contenidos entre jugadores, pedagógicos y de conocimiento en afinar las estrategias de enseñanza efectiva, Mishra \& Koehler extendieron este concepto adicionando cuatro dimensiones teóricas. En este sentido los investigadores plantearon el modelo TPCN (tecnológico, pedagógico, contenido y conocimiento), el cual facilita los niveles de integración tecnológica, teórica, pedagógica y metodológica, mientras que ayuda a diseñar 
estratégicas pedagógicas para la enseñanza con tecnología. Una adaptación al modelo TPCN fue realizado por Lee \& Tsai (2010), al cual integraron la dimensión web. En este sentido, ellos trataron de presentar un marco que soportara el entendimiento del conocimiento Web en los contenidos tecnológicos y pedagógicos (TPNC-W), mientras integraban la tecnología WEB dentro de las prácticas pedagógicas.

Ellis, Goodyear, Prosser \& O’Hara (2006) discutieron que los docentes en muchas oportunidades transfieren parte de sus mejores prácticas aprendidas en la enseñanza de cursos de educación a distancia (tales como sus hábitos de gestión y organizacionales, la atención a los detalles, y la anticipación a la falta de entendimiento de los estudiantes) a los ambientes de enseñanza cara a cara tradicionales. Asimismo, Lowes (2008) presentó que los cursos de enseñanza en línea han estimulado a los docentes al uso de prácticas pedagógicas innovadoras fomentó enormemente la participación de los estudiantes, motivó la reflexión e hizo que algunos docentes crearan empatía y sensibilidad a las necesidades de los estudiantes.

Por su lado, la Unesco (2008) desarrolló unos estándares de competencias en TIC para docentes el cual tiene como finalidad mejorar la práctica de los docentes en todas las áreas de su desempeño profesional, combinando las competencias en TIC con innovaciones en la pedagogía, el plan de estudios (currículo) y la organización escolar. El objetivo general de este proyecto es mejorar la práctica de los docentes y la calidad del sistema educativo con la finalidad de contribuir al desarrollo económico y social, por consiguiente para lograr todo esto es indispensable que el docente adquiera las competencias que se presentan en la próxima tabla: 
Tabla N..$^{\circ} 1$

Los estándares de competencias en TIC para docentes

\begin{tabular}{|c|c|c|c|}
\hline $\begin{array}{c}\text { Política } \\
\text { y } \\
\text { visión }\end{array}$ & $\begin{array}{c}\text { Nociones } \\
\text { básicas } \\
\text { de TIC }\end{array}$ & $\begin{array}{c}\text { Profundización } \\
\text { del } \\
\text { conocimiento }\end{array}$ & $\begin{array}{c}\text { Generación } \\
\text { de } \\
\text { conocimiento }\end{array}$ \\
\hline $\begin{array}{c}\text { Plan de estudios } \\
\text { y evaluación }\end{array}$ & $\begin{array}{c}\text { Conocimientos } \\
\text { básicos }\end{array}$ & $\begin{array}{c}\text { Aplicación del } \\
\text { conocimiento }\end{array}$ & $\begin{array}{c}\text { Competencias } \\
\text { del siglo XXI }\end{array}$ \\
\hline Pedagogía & $\begin{array}{c}\text { Integrar las } \\
\text { TIC }\end{array}$ & $\begin{array}{c}\text { Solución de pro- } \\
\text { blemas complejos }\end{array}$ & Autogestión \\
\hline TIC & $\begin{array}{c}\text { Herramientas } \\
\text { básicas }\end{array}$ & $\begin{array}{c}\text { Herramientas } \\
\text { complejas }\end{array}$ & $\begin{array}{c}\text { Tecnología } \\
\text { generalizada }\end{array}$ \\
\hline $\begin{array}{c}\text { Organización y } \\
\text { administración }\end{array}$ & $\begin{array}{c}\text { Aula de clase } \\
\text { estándar }\end{array}$ & $\begin{array}{c}\text { Grupos } \\
\text { colaborativos }\end{array}$ & $\begin{array}{c}\text { Organizaciones } \\
\text { de aprendizaje }\end{array}$ \\
\hline $\begin{array}{c}\text { Formación } \\
\text { profesional } \\
\text { de docentes }\end{array}$ & $\begin{array}{c}\text { Alfabetismo } \\
\text { en TIC }\end{array}$ & Gestión y guía & $\begin{array}{c}\text { El docente } \\
\text { modelo de } \\
\text { educando }\end{array}$ \\
\hline
\end{tabular}

Fuente: Unesco (2008). Estándares de competencias en TIC para docentes.

La Universidad Abierta a Distancia (UNAD) de Colombia ha creado un modelo de competencias docentes que fue presentado por Herrera (2008) el cual consta de once competencias fundamentales. Estas competencias son las siguientes:

1) Competencia cognitiva

2) Competencia socio-afectiva

3) Competencia comunicativa

4) Competencia pedagógica

5) Competencia didáctica

6) Competencia metodológica

7) Competencia tecnológica

8) Competencia disciplinar

9) Competencia investigativa

10) Competencia comunitaria

11) Competencia de trabajo colaborativo 
Con la implementación de este modelo de competencias, la UNAD busca que cada uno de sus miembros del cuerpo académico pueda desarrollar sus actividades manteniendo una autonomía intelectual soportada por un pensamiento heurístico, flexible, complejo, hermenéutico, deconstructor-reconstructor de paradigmas, político, interdisciplinario y en red.

Mediante un estudio desarrollado por Abdous (2011) se presenta la propuesta de un patrón con la finalidad de estructurar las competencias docentes mediante un proceso que integra tres fases no lineales:

1) Antes: planificación y diseño.

2) Durante: facilitación, interacción y retroalimentación.

3) Después: reflexión.

El objetivo principal de este estudio tiene tres vertientes: proponer un patrón sistemático y práctico capaz de clarificar las competencias asociadas a la enseñanza en línea; permitir a los docentes crear y facilitar oportunidades efectivas de aprendizaje y ayudar a las instituciones de educación superior a establecer un programa de desarrollo docente comprensible y efectivo de educación en línea.

En resumen, con mínimas variaciones, el consenso de los investigadores en el temas de las competencias docentes en el uso de las TIC aparenta querer incluir los cuatro roles y competencias, que son pedagógica, gestión, social y técnico. La dimensión pedagógica considera la preparación del contexto, la interacción, la colaboración y las habilidades y competencias, mientras que la dimensión de gestión se enfoca en los elementos logísticos y de disposición. La dimensión social se enfoca en la construcción de la comunidad, la interacción y la colaboración, mientras que la dimensión técnica se centra en las capacidades del uso del computador y la gestión del curso. Sin embargo, es difícil mapear estos roles ante las fases de experiencia de la educación en línea. 


\section{Conclusiones. Una visión sistémica para superar los obstáculos que no han permitido una mayor expansión de las TIC}

La integración de las TIC en la educación superior es inevitable; no es algo por hacer: es una realidad. En los próximos años el empuje será sobre el uso de las TIC para fortalecer el sistema y el modo de facilitar el aprendizaje a distancia. Tanto las instituciones gubernamentales como las instituciones de educación superior deberán identificar el rol específico de las TIC para la mejora y crecimiento de la educación a nivel mundial, a la vez mejorando las capacidades de investigación y proveyendo una estructura adecuada a través de la construcción de capacidades específicas. En la actualidad, se reconoce la importancia de la educación basada en la tecnología, no obstante existen todavía una serie de factores y obstáculos que han impedido una mayor integración de las TIC en la educación superior. Algunas de las causas más relevantes que se han identificado podrían ser la escasa formación entre los profesionales de la enseñanza, la falta de financiamiento para la adquisición de la tecnología, el deficiente desarrollo de políticas públicas específicas en apoyo a este proceso, y la poca motivación entre los profesores en adoptar las TIC como herramientas de enseñanza (Starr, 2001).

La amplia adopción de las TIC demanda formas de pensar y habilidades que acepten el cambio. En este sentido, y aunque dentro de las instituciones de educación superior de los países desarrollados se han creado marcos políticos que amparan el uso de las tecnologías dentro del proceso de aprendizaje, todavía existen muchas controversias sobre el equilibrio de la investigación académica a largo plazo con los proyectos de transferencia de tecnología a corto plazo.

Los docentes tienen que asumir un proceso de desarrollo profesional continuo para implementar el uso de la tecnología en el proceso de enseñanza. En este sentido, los profesores tienen que 
estar preparados para hacer uso de las posibilidades que ofrecen las TIC, tales como diferentes contextos de aprendizaje, un enfoque hacia el estudiante, presentándoles diferentes tipos de interacción, diferentes grados de control en el auto-aprendizaje, adaptándose a sus intereses personales, promoviendo las tareas de colaboración y el desarrollo de autonomía en su trabajo y estudio (Toro \& Joshi, 2012).

A pesar de todos los recursos financieros que han sido y continúan siendo dirigidos para apoyar y adquirir tecnologías de aprendizaje, los resultados han sido generalmente decepcionantes (Selwyn, 2007). Los docentes están dispuestos a utilizar la tecnología, pero carecen de los conocimientos técnicos, la habilidad y la experiencia (Buczynski y Mall, 2010).

El mundo académico es altamente conservador, y las instituciones de educación superior que no estén activamente involucradas en integrar el uso de la tecnología dentro de su proceso de enseñanza podrán ver mermar su población universitaria en los próximos años. Aunque los proveedores de la tecnología han convencido a los tomadores de decisiones en la educación superior para adoptar esta tendencia, la realidad de lo que realmente ocurre es bastante diferente.

Según Schneckenberg (2009), los talleres y los hechos han demostrado que los docentes tienen grandes debilidades en la eficacia y el uso de las TIC. Lamentablemente, las tecnologías van y vienen y son utilizadas por unos pocos elegidos. Sin lugar a dudas, una poderosa razón para esto es que la integración exitosa y sostenible de la tecnología en las actividades de enseñanza y aprendizaje demandan cuestionar muchas creencias profundamente arraigadas, el cambio de las prácticas establecidas desde hace tiempo, y el fomento de nuevas formas de pensar en una institución (Bates, 2000: 42-43; Cook- Sather , 2001: 128 ). 
No se puede hablar de enseñanza a través de los medios tecnológicos sin lograr una adhesión de parte de los docentes (antiguos proveedores del conocimiento, hoy facilitadores del mismo). La sociedad actual no demanda una educación encasillada en los viejos paradigmas; hoy, porque el mundo ha cambiado se necesita una educación abierta, flexible, pero de calidad.

Se debe reconocer que la resistencia al cambio no es solo una actitud de los docentes. Las bajas competencias docentes también es un reflejo - y se debe en gran medida - a una falta de planificación y voluntad de los que administran las instituciones de educación superior.

\section{Referencias bibliográficas}

Abdous, M. (2011). A process - oriented framework for acquiring online teaching competencies. Journal of Computing in Higher Education, 23: 60-77. doi: 10.1007/s12528-010-9040-5.

Abdous, M. \& Yoshimura, M. (2010). Learner outcomes and satisfaction: A comparison of live videostreamed instruction, satellite broadcast instruction, and face-to-face instruction. Computers \& Education, 55(2), 733-741.

Abel, R. (2005). Achieving Success in Internet-Supported Learning in Higher Education: Case Studies Illuminate Success Factors, Challenges, and Future Directions. Recuperado de http://www.ahec.org/IsL_ orig_study.html

Adell, J. (2004). Internet en el aula: Las WebQuest. [Revista Electrónica de Tecnología Educativa, 17]. Recuperado de http://edutec. rediris.es/Revelec2/revelec17/adell_16a.htm>

Aguilar, M. A. \& Cepeda, B. (2005). Pisa para docentes. La evaluación como oportunidad de aprendizaje. México: Comisión Nacional de Libros de Texto Gratuitos. Recuperado de http://www.edu cacionbc.edu.mx/departamentos/evaluacion/descargas/ Archivos/PISA_docentes.pdf 
Nuevos roles de los docentes en la educación superior: hacia un nuevo perfil y modelo de competencias con integración de las TIC

Alarcón, R. (2012). La internacionalización de la educación a distancia y su alcance a las zonas excluidas dentro del territorio nacional. En M. Morocho \& C. Rama (Eds:). Las nuevas fronteras de la educación a distancia. Ecuador, Loja: Ediloja.

Albornoz, M. (2002). La universidad ante la innovación. (Ponencia). En Casas, M \& Stojanovic, L. (2013). Innovación en la universidad iberoamericana. Revista de la Universidad y Sociedad del Conocimiento (RUSC), 10(1), 61-74.

Amirault, R., \& Visser, Y. (2009). The university in periods of technological change: A historically grounded perspective. Journal of Computing in Higher Education, 21(1), 62-79.

Barker, P. (2002). On Being an Online Tutor. Innovations in Education and Teaching International, 39(1), 3-13.

Bates, A. (2000). Managing Technological Change: Strategies for College and University Leaders. San Francisco: Jossey-Bass.

Bawane, J., \& Spector, J. M. (2009). Prioritization of online instructor roles: Implications for competency-based teacher education programs. Distance Education, 30(3), 383-397.

Berge, Z. L. (1995). Facilitating computer conferencing: Recommendations from the field. Educational Technology, 35(1), 22-30.

Briggs, S. (2005). Changing roles and competencies of academics. Active Learning in Higher Education, 6(3), 256-268.

Buczynski, J. \& Mall, R. (2010). Bridging the Gap. Internet Reference Services Quarterly, 15: 87-96, 2010. doi: 10.1080/1087530100 3757211.

Casas Armengol, M. (2005). Nueva universidad ante la sociedad del conocimiento. Revista de Universidad y Sociedad del Conocimiento (RUSC), 2(2), 1-18. Recuperado de www.uoc.edu/ rusc/2/2/dt/esp/casas.pdf. 
Chiavenato, I. (2002). Gestión del talento bumano. Bogotá: McGrawHill Interamericana.

Comisión de las Comunidades Europeas. (2007). Competencias clave para el aprendizaje permanente. Recuperado de http://www.crue. org/export/sites/Crue/procbolonia/documentos/antece dentes/9._Competencias_clave_para_aprendizaje_perma nente.pdf

Cook-Sather, A. (2001). Unrolling roles in techno-pedagogy: Toward new forms of collaboration in traditional college settings. Innovative Higher Education, 26(2):122-140.

Coppola, N. W., Hiltz, S. R., \& Rotter, N. G. (2002). Becoming a virtual professor: Pedagogical roles and asynchronous learning networks. Journal of Management Information Systems, 18(4), 169-189.

Competencias Informáticas e Informacionales y Red de Bibliotecas Universitarias (2009). Competencias informáticas e informacionales en los estudios de grado. Recuperado de http://www.rebiun.org/ doc/documento_competencias_informaticas.pdf.

Dirección General de Ordenación y Evaluación Educativa de Andalucía. (2012). Guía sobre buenas prácticas docentes para el desarrollo en el aula de las competencias básicas del alumnado. Recuperado de http://www.juntadeandalucia.es/educacion/ portal/com/bin/Contenidos/OEE/planesyprogramas/ documentos_buenas_practicas/1357905078413_guia.pdf

Ellis, R. A., Goodyear, P., Prosser, M. \& O’Hara, A. (2006). How and what university students learn through online and faceto-face discussion: Conceptions, intentions and approaches. Journal of Computer Assisted Learning, 22, 244-256.

Escudero, J. (2006). La formación del profesorado y la garantía del derecho a una buena educación para todos. En J. Escudero \& A. Luis. La formación del profesorado y la mejora de la 
Nuevos roles de los docentes en la educación superior: hacia un nuevo perfil y modelo de competencias con integración de las TIC

educación para todos: políticas y prácticas. (pp. 21-51). Barcelona: Octaedro.

Fainholc, B. (2002). La calidad en la educación a distancia continúa siendo un tema muy complejo. Recuperado de www.um.es/ead/ red/12/fainholc.pdf

Gallart, M. y Jacinto, C. (1995). Competencias laborales: tema clave en la articulación educación trabajo. Boletín de la Red Latinoamericana de Educación y trabajo, 6, 2, s. p.

Goodyear, P., Salmon, G., \& Spector, J. M. (2001). Competences for online teaching: A special report. Educational Technology Research and Development, 49(1), 65-72.

Harasim, L.; et al. (2006). Redes de aprendizaje. Barcelona: Editorial Gedisa.

Harasim, L. (2006). A history of E-learning: Shift happened. In J. Weiss, J. Nolan, J. Hunsinger, \& P. Trifonas (Eds.). The international handbook of virtual learning environments, (pp. 59-94). Netherlands: Springer.

Hayes, R. (1985). Strategic planning forward in reverse. Harvard Business Review, 63, 111-119.

Heather, J. (2012). Faculty development programs: the frequency and variety of professional development programs available to online instructors. Journal of asynchronous learning networks, 16: $5.87-106$.

Hellriegel, D. \& Slocum, J. (2005). Comportamiento Organizacional. México: Thomson Editores.

Herrera, G. (2008). Competencias del cuerpo Académico de la Universidad Nacional Abierta y a Distancia. Recuperado de http://www.uapa. edu.do/docs/revista/Enero-Diciembre2008.pdf 
Imbernon, F., Silva, P. \& Guzmán, C. (2011). Competencias en los procesos de enseñanza-aprendizaje virtual y semipresencial. Comunicar, 36, 107-114. (doi: 10.3916/C36-2011-03-01).

Kanuka, H \& Kelland, J. (2008). Has e-Learning Delivered on its Promises? Expert Opinion on the Impact of e-Learning in Higher Education. Canadian Journal of Higher Education Revue, 38(1), 45-65.

Katz, J. \& Hilber, M. (2003). Los caminos hacia una sociedad de la información en América Latina y el Caribe. Recuperado de www.eclac.cl/publicaciones/DesarrolloProductivo/1/LCG 2195Rev1P/lcg2195e2.pdf

Kennedy, D. \& McNaught, C. (1997). Design elements for interactive multimedia. Australian Journal of Educational Technology, 13(1), 1-22.

Larraz, V., Sánchez, J., Casalprim, M. Saz, A. (2013). El entorno virtual de la Univeritat d'Andorra. La visión del profesorado. Recuperado de http://www.um.es/ead/red/36/larraz.pdf.

Laurillard, D. (2008). Technology enhanced learning as a tool for pedagogical innovation. Journal of Philosophy of Education, 42(3/4), 521-533.

Lee, M.-H., \& Tsai, C.-C. (2010). Exploring teachers' perceived self efficacy and technological pedagogical content knowledge with respect to educational use of the World Wide Web. Instructional Science, 38(1), 1-21.

Lentell, H. (2003). The Importance of the Tutor in Open and Distance Learning. En: A. Tait \& R. Mills (Eds.). Rethinking Learner Support In Distance Education. (pp. 64-76). London: Routledge Falmer.

LevyLevoyer, C. (2002). Gestión de las competencias, cómo analizarlas, cómo evaluarlas, cómo desarrollarlas. Barcelona: Gestión 2000. 
Nuevos roles de los docentes en la educación superior: hacia un nuevo perfil y modelo de competencias con integración de las TIC

Lowes, S. (2008). Online teaching and classroom change: The trans-classroom teacher in the age of the internet. Innovate. Recuperado de http://innovateonline.info/pdf/vol4_issue3/Online_ Teaching_and_Classroom_Change-_The_Trans-Classroom _Teacher_in_the_Age_of_the_Internet.pdf.

McShane, K. (2004). Integrating face-to-face and online teaching: Academics' role concept and teaching choices. Teaching in Higher Education, 9(1), 3-16.

Means, B., Toyama, Y., Murphy, R., Bakia, M., \& Jones, K. (2009). Evaluation of evidence based practices in online learning: $A$ metaanalysis and review of online learning studies. Recuperado de http://www.ed.gov/rschstat/eval/tech/evidence-basedpractices/finalreport.pdf.

Méndez-Estrada, V. \& Monge, J. (2006). Las TIC en un entorno latinoamericano de educación a distancia: la experiencia de la UNED de Costa Rica. Recuperado de http://www.um.es/ead/red/15/.

Mishra, P., \& Koehler, M. (2006). Technological pedagogical content knowledge: A framework for teacher knowledge. Teachers College Record, 108(6), 1017-1054.

Montmollin. 1984. L'intelligence de la tache. Lang, Berna.

Murcia, J. (2004). Redes del saber: investigación virtual, proceso educativo y autoformación integra. Bogotá: Editorial Magisterio.

Navarro, R. (2009). Las nuevas tecnologías para el aprendizaje: estado del arte. En: J. Vales. Nuevas tecnologías para el aprendizaje. (pp. 15-26). México: Pearson Educación.

Oliver, R. (2000). Creating Meaningful Contexts for Learning in Web-based Settings. Proceedings of Open Learning 2000. (pp 53-62). Brisbane: Learning Network.

Oliver, R. \& Short, G. (1996). The Western Australian Telecentres Network: A model for enhancing access to education 
and training in rural areas. International Journal of Educational Telecommunications, 2(4), 311-328

Organización de los Naciones Unidas para la Educación, la Ciencia y la Cultura. (2008). Estándares de competencias en tic para docentes. Recuperado de http://www.eduteka.org/pdfdir/ UNESCOEstandaresDocentes.pdf

Organización de los Naciones Unidas para la Educación, la Ciencia y la Cultura (2009). Conferencia Mundial sobre la Educación Superior 2009: La nueva dinámica de la educación superior y la investigación para el cambio social y el desarrollo. Recuperado de http://www.unesco.org/education/WCHE2009/comu nicado_es.pdf

Padula Perkins, J. E. (2003). Una introducción a la educación a distancia. Buenos Aires: Fondo de Cultura Económica.

Padula, J. (2004). Contigo en la distancia. El rol del tutor en la educación no presencial. Cátedra UNESCO en la educación a distancia, Recuperado de http://www.uned.es/catedraunesco-ead/ Docs_Publicued/pbc08/rol_bened.htm

Palomo, R., Ruis, J. \& Sánchez, J. (2006). Las TIC como agentes de innovación educativa. Sevilla. Junta de Andalucía-Consejería de Educación.

Parsad, B., \& Lewis, L. (2008). Distance education at degree-granting postsecondary institutions: 200607. Recuperado de http:/ / nces.ed. gov/pubSearch/pubsinfo.asp?pubid $=2009044$.

Paulsen, M. F. (1995). Moderating educational computer conferences. In Z. L. Berge \& M. P. Collins (Eds.). Computer mediated communication and the online classroom. Vol. III: Distance Learning (pp. 81-89). New Jersey: Hampton Press.

Pereda, S. y Berrocal, F. (2001). Gestión de recursos humanos por competencia. Madrid: Centro de Estudios Ramón Areces. 
Nuevos roles de los docentes en la educación superior: hacia un nuevo perfil y modelo de competencias con integración de las TIC

Prieto (1997). La gestión de las competencias. Barcelona: Ediciones Gestión 2000.

Rama, C. (2009). La tendencia a la masificación de la cobertura de la educación superior en América Latina. Revista Iberoamericana de Educación, 50, 173-195. Recuperado de http;//www.rieoie.org/rie50a09.pdf.

Rama, C. (2012). La reforma de la virtualización de la universidad: el nacimiento de la educación digital. Recuperado de http://www.virtualeduca.org/documentos/observatorio/ libro_la-reforma-de-la-virtualizacion-de-la-universidadclaudio-rama-udg-2012.pdf

Reigeluth, C. (2012). Instructional Theory and Technology for the New Paradigm of Education. Recuperado de http://www.um.es/ $\mathrm{ead} / \mathrm{red} / 32$

Ryan, S., Scott, B., Freeman, H., \& Patel, D. (2000). The Virtual University: The Internet and Resource-Based Learning. London: Kogan page Limited.

Sangrà, a. (2002): La calidad en las experiencias virtuales de educación superior. Barcelona: UOC. Recuperado el 26 de junio de 2013 en: www.uoc.edu/web/esp/art/uoc/0106024/sangra.html (consulta junio de 2009).

Selwyn, N. (2007). The use of computer technology in university teaching and learning: A critical perspective. Journal of Computer Assisted Learning, 23(2):83-94.

Schneckenberg, D. (2009). Understanding the real barriers to technology-enhanced innovation in higher education. Educational Research, 51(4):411-424.

Shulman, L. S. (1987). Knowledge and teaching: Foundations of the new reform. Harvard Educational Review, 57(1), 1-22. 
Silvio, J. (2006). Hacia una educación virtual de calidad, pero con equidad y pertinencia. Revista de Universidad y Sociedad del Conocimiento Vol. 3 - N. 1 / Abril de 2006, Recuperado el 26 de junio de 2013 en: http:/ /www.uoc.edu/rusc/3/1/dt/esp/ silvio.pdf

Simpson, O. (2002). Supporting Students in Open and Distance Learning. London: Kogan.

Starr, L. (2001). Same time this year. [En línea]. Recuperado de http://www.education-world.com/a_tech/tech075.shtml

Suarez, J. M. \& Anaya, D. (2004). Educación a distancia y presencia: diferencias en los componentes cognitivo y motivacionales de estudiantes universitarios. Revista Iberoamericana de Educación a distancia, 7(1/2), 65-75.

Tapscott, D., \& Williams, A. D. (2010). Innovating the 21st-century university: It's time! EDUCAUSE Quarterly, 45(1), 16-29. Recuperado de http://www.educause.edu/EDUCAUSE? Review/EDUCAUSEReviewMagazineVolume45/Innova tingthe21stCenturyUniver/195370.

Tello, J. (2003). Competencia informática del profesorado de Infantily Primaria en Huelva y su incidencia en la práctica docente. En: Gutiérrez Porlán, I. (2011). Competencias del profesorado universitario en relación al uso de las tecnologías de la información y la comunicación: análisis de la situación en España y propuesta de un modelo de formación. Recuperado de http://www.tdx.cat/bitstream/handle/ 0803/52835/tesiscompleta_gutierrez_porlan_isabel.pdf? sequence $=1$.

Tello, J. y Aguaded, J. (2009). Desarrollo profesional docente ante los nuevos retos de las tecnologías de la información y la comunicación en los centros educativos. Recuperado de http://www.sav.us.es/pixelbit/ pixelbit/articulos/n34/n34.html. 
Nuevos roles de los docentes en la educación superior: hacia un nuevo perfil y modelo de competencias con integración de las TIC

Tünnermann, C. (2000). Universidad y Sociedad: balance bistórico y perspectivas desde Latinoamérica. Caracas: Universidad Central de Venezuela.

Uñantes, G., Reynoso, E. \& Brescia, M. (2001). E-learning: cambiando paradigmas en capacitación. Recuperado de www.contenidos. com/bitacora/opinion.php3?=hoy2001-10-25.

Vales, J. (2009). Nuevas tecnologias para el aprendizaje. México: Pearson Educación

Vaughan, N. (2007). Perspectives on blended learning in higher education. International Journal on E-Learning, 6(1), 81-94.

Waheed Khan, A. (2008). Estándares de competencias en tic para docentes. Recuperado de http:/ / www.eduteka.org/pdfdir/ UNESCOEstandaresDocentes.pdf

\section{Luis José Camacho Rivas}

Es mentor y profesor en Empire State College, USA; Profesor Berkeley College, USA. Ha sido profesor y coordinador de Programas y Proyectos en el Instituto Tecnológico de Santo Domingo; profesor de la Universidad Pedro Henriquez Ureña, entre otras universidades. Investigador, consultor, director y asesor de empresas; conferencista y diseñador de múltiples proyectos educativos a nivel graduado y postgraduado. 
Es licenciado en Administración de Empresas, Universidad Católica de Santo Domingo; máster en Mercadeo, Instituto Tecnológico de Santo Domingo; especialista en Gerencia de la Industria Textil, China Textile Institute (CTI); doctor en Educación con Concentración en Tecnología Instruccional y Educación a Distancia (candidato), Nova Southeastern University. Posee además una amplia formación a través de cursos expertos, diplomados y seminarios celebrados en los Estados Unidos, China, Italia, México, Colombia, Cuba y Costa Rica.

Su línea de investigación en educación superior está concentrada en educación a distancia y virtual, competencias profesionales, competencias docentes, competencias basadas en TIC. En el ámbito profesional ha realizado investigaciones sobre mercados emergentes y gerencial internacional.

Email: luis.camacho@esc.edu

Recibido: 29/05/2014 Aprobado: 25/10/2014 